\title{
Modeling complexity in pathologist workload measurement: the Automatable Activity-Based Approach to Complexity Unit Scoring (AABACUS)
}

Carol C Cheung ${ }^{1,2}$, Emina E Torlakovic ${ }^{2,3}$, Hung Chow ${ }^{4}$, Dale C Snover ${ }^{5}$ and Sylvia L Asa ${ }^{1,2}$

${ }^{1}$ Department of Pathology, Laboratory Medicine Program, University Health Network, Toronto, ON, Canada; ${ }^{2}$ Department of Laboratory Medicine and Pathobiology, Faculty of Medicine, University of Toronto, Toronto, ON, Canada; ${ }^{3}$ Department of Laboratory Hematology, Laboratory Medicine Program, University Health Network, Toronto, ON, Canada; ${ }^{4}$ Laboratory Information Support Systems, Laboratory Medicine Program, University Health Network, Toronto, ON, Canada and ${ }^{5}$ Department of Pathology, Fairview Southdale Hospital, Edina, MN, USA

Pathologists provide diagnoses relevant to the disease state of the patient and identify specific tissue characteristics relevant to response to therapy and prognosis. As personalized medicine evolves, there is a trend for increased demand of tissue-derived parameters. Pathologists perform increasingly complex analyses on the same 'cases'. Traditional methods of workload assessment and reimbursement, based on number of cases sometimes with a modifier (eg, the relative value unit (RVU) system used in the United States), often grossly underestimate the amount of work needed for complex cases and may overvalue simple, small biopsy cases. We describe a new approach to pathologist workload measurement that aligns with this new practice paradigm. Our multisite institution with geographically diverse partner institutions has developed the Automatable Activity-Based Approach to Complexity Unit Scoring (AABACUS) model that captures pathologists' clinical activities from parameters documented in departmental laboratory information systems (LISs). The model's algorithm includes: 'capture', 'export', 'identify', 'count', 'score', 'attribute', 'filter', and 'assess filtered results'. Captured data include specimen acquisition, handling, analysis, and reporting activities. Activities were counted and complexity units (CUs) generated using a complexity factor for each activity. CUs were compared between institutions, practice groups, and practice types and evaluated over a 5year period (2008-2012). The annual load of a clinical service pathologist, irrespective of subspecialty, was $\sim 40000$ CUs using relative benchmarking. The model detected changing practice patterns and was appropriate for monitoring clinical workload for anatomical pathology, neuropathology, and hematopathology in academic and community settings, and encompassing subspecialty and generalist practices. AABACUS is objective, can be integrated with an LIS and automated, is reproducible, backwards compatible, and future adaptable. It can be applied as a robust decision support tool for the assessment of overall and targeted staffing needs as well as utilization analyses for resource allocation.

Modern Pathology (2015) 28, 324-339; doi:10.1038/modpathol.2014.123; published online 12 September 2014

The work of pathologists has historically evolved into complex types of practices, recently at least

Correspondence: Dr CC Cheung, MD, PhD, Department of Pathology, University Health Network, 200 Elizabeth Street, 11th Floor, Eaton Wing, Toronto, ON M5G 2C4, Canada.

E-mail: c.cheung@utoronto.ca

Received 24 March 2014; revised 7 July 2014; accepted 8 July 2014; published online 12 September 2014 partly due to the increasing complexity of the WHO classification of diseases as well as the increasing role of tissue-based parameters in patient care. ${ }^{1}$ The advent of personalized medicine has shifted the paradigms of medical practice including pathology. ${ }^{2,3}$ Today's treatment decisions, especially in fields like oncology, are becoming increasingly individualized and rely on numerous specific parameters derived from excised human tissue 
including exact size of lesions, patterns of growth, surgical margins, degree and type of differentiation, presence and number of precursor lesions, immunophenotyping, proteomic and genomic profiling to determine eligibility for targeted therapy, genetic predisposition, and other individual, family, and societal risk factors.

Pathologists are required to perform multiparameter analysis of patient-derived materials and consequently issue reports of increasing complexity so that the right patient receives the right treatment at the right time. Information from pathology reports also feeds into organizational, regional, and/or national databases to facilitate the planning of health care. ${ }^{4}$ As suppliers of knowledge that is critical to medical care, pathologists must ensure that appropriate expertise and manpower resources are in place to safely accommodate the information demand that drives personalized medicine.

Although in many organizations, pathologist staffing is based entirely on fee-for-service income, many pathologists have salary or contract employment with large organizations or academic partnerships in academic medical centers. In light of the transformation of pathologist duties and changing workloads, a critical question for institutions with respect to manpower planning is: how many pathologists do we need? ${ }^{5-8}$ The answer to that question depends entirely on what pathologists are required to do. There is a clear need to define this workload and, once defined, to effectively measure it. As practices vary both between institutions and between subspecialties, there is a need for a tool that can measure pathologist workload irrespective of practice type (ie, general versus subspecialty) or differing institutional expectations. ${ }^{5,6}$ In academic centers, pathologist duties may consist of varying proportions of the archetypical Clinical/Research/ Education spheres (Figure 1); ${ }^{9}$ measures of workload can be obtained for teaching hours, course development, and research activities; however, there is a need for a pathologist workload measurement model that is reflective of the clinical aspects of a pathologist's work and particularly on one component of clinical work, namely the work related to the issuing of a diagnostic medical report (Figure 2). As seen in Figure 2, the work of pathologists is complex; the Automatable Activity-Based Approach to Complexity Unit Scoring (AABACUS) is designed to measure the work done in generating diagnostic medical reports. It is fortunate that many laboratory information systems (LISs) collect parameters that can be conveniently used to capture this type of pathologists' work.

The inability to define or design a concept of capturing actual work performed is a stumbling block in pathologist workload measurement tools. Currently available models depend on manual entry of workload-specific parameters by pathologists that are based on 'specimen type' or that assign relative weightings based on those derived from 'billing'

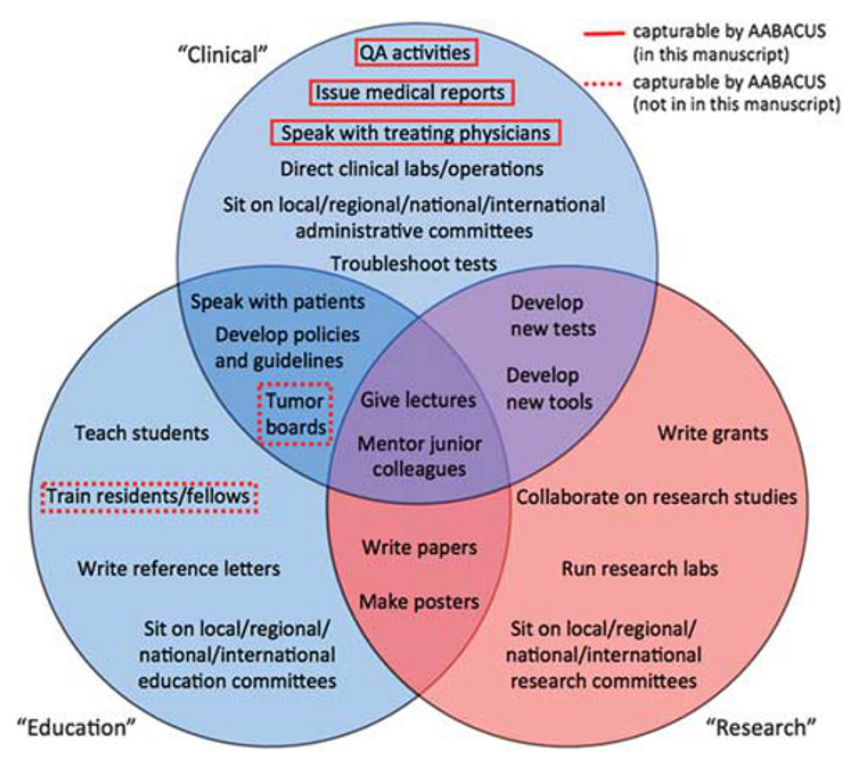

Figure 1 The Clinical/Education/Research spheres of 'pathologist work'.

Specimen Acquisition Activities e.g. FNAs by pathologists...etc..

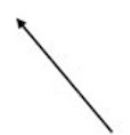

Issue medical reports

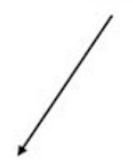

Analysis Activities

e.g. Morphologic analysis of tissue sections, cytologic preparations, reportable parameters of synoptic worksheets...etc.

Figure 2 Classes of clinical activities that contribute to the issuing of a diagnostic medical report in pathology, with examples.

models. ${ }^{10-16}$ Some models that depend on manual entry or coding by pathologists to capture the parameters used to measure workload may lack objectivity and reproducibility because they involve issues of interpretation and compliance. Other manual entry modes, like the relative value unit (RVU) system used in the United States, avoid this 'problem' as there is no allowed variance in how cases are coded, that is, excisional breast biopsies without identified margins are coded as 88305 regardless of size, complexity of diagnosis, and so on. Models that take the approach of measuring workload based on specimen/tissue type and the 'work and effort' that a particular specimen type is felt to entail relative to other specimen types lack granularity because depending on circumstances, the same specimen type often requires differing amounts of pathologist work. If 'billing' tools form 
the basis for assigning relative weights for pathologist workload measurement, then this reflects only what a payer is willing to pay for the work in a particular economic environment at a particular point in time. This 'price' can arbitrarily change with shifts in either political or economic circumstances without any actual changes in practice and/or pathologist workload, and this may be detrimental to either the payer or payee. ${ }^{17}$ To properly plan manpower needs, the approach used to measure workload must measure work and not its potential value to a payer.

We defined a workload measurement model that allows for the capture of workload parameters in a manner that is not pathologist dependent, that is activity based rather than specimen type based, and that assigns relative weightings based on time rather than arbitrary value to a payer. We present here the AABACUS model as a robust decision-support tool for laboratory management of a multisite pathology laboratory with geographically diverse partner institutions that must accommodate both community-based generalist and subspecialty-based academic staffing needs in anatomical pathology (including cytopathology), neuropathology, and hematopathology.

\section{Materials and methods}

The concept of AABACUS is based on the framework of capturing pathologist clinical activities independent of the specimen type or subspecialty. From a practical perspective, AABACUS takes information from laboratory information systems and converts it into counts of pathologist clinical activities that are then translated into pathologist clinical workload units. The overall algorithm for the execution of the calculations is as follows: capture, export, identify, count, score, attribute, filter, and assess filtered results (Figure 3).

\section{Capture}

LISs are designed to capture various types of data; some of these data are informative for work performed by pathologists and some are not. All data used by AABACUS are those that were

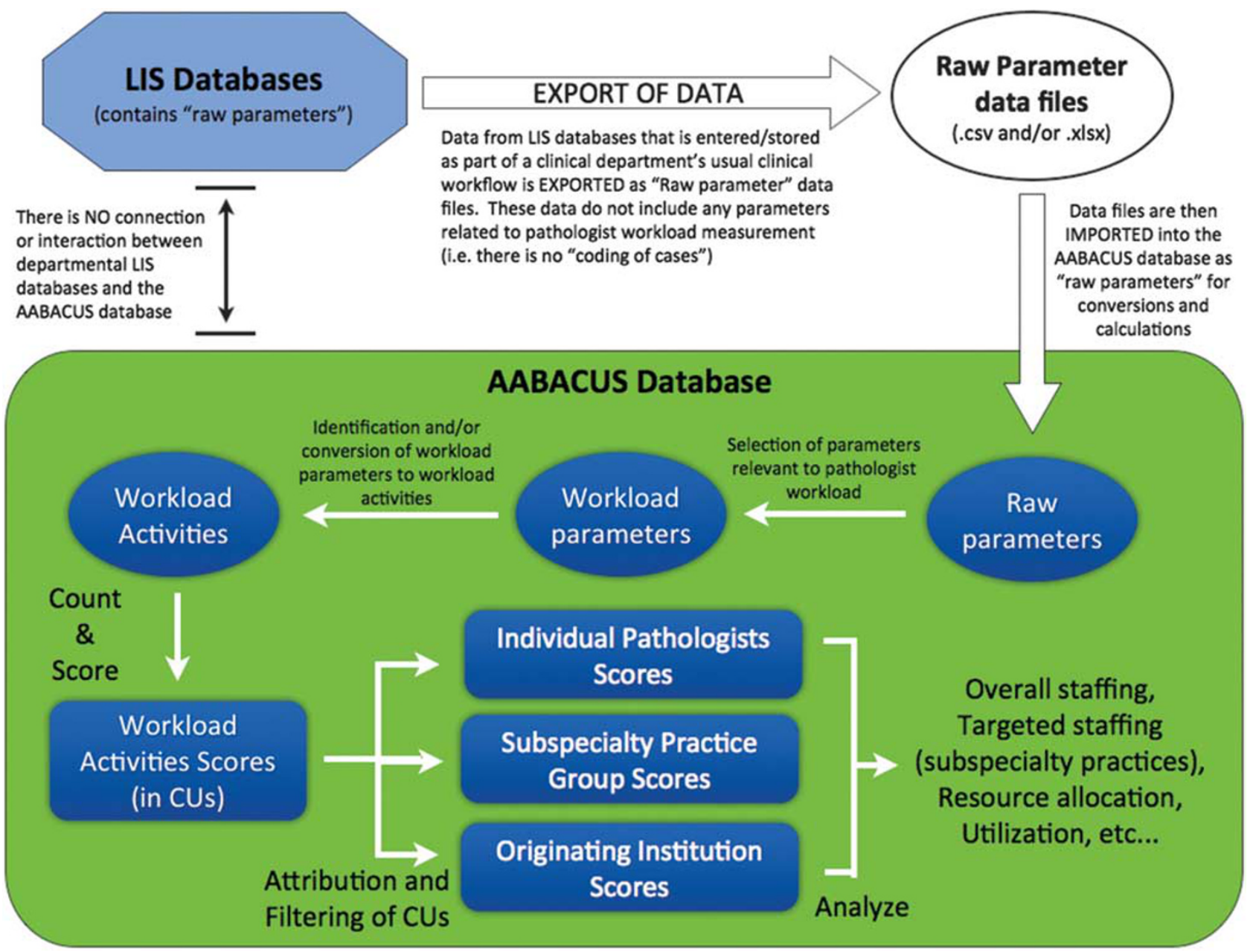

Figure 3 Overview of AABACUS. 
originally captured and stored in departmental LISs as a part of usual clinical workflow; we have defined these as 'raw parameters'. Examples of raw parameters include: number of blocks/slides, number of special stains, number of levels, presence of completed synoptic reporting templates, issued addenda and the person who issued them, frozen sections cut, frozen section reports and the person who issued them, number of specimens, QA entries and person who entered the QA entries, gross dictations and the person who performed and dictated the gross, as well as additional persons who reviewed but did not actually dictate the gross description. The numbers of levels and/or serial sections per slide were defined by protocol order with uniformity of practice across all institutional laboratories. Raw parameters did not include parameters specifically related to pathologist workload measurement because we do not 'code' our cases for either workload or billing purposes. No additional capture of data above and beyond what is normally recorded as part of usual clinical workflow was required by AABACUS.

\section{Export}

Raw parameters that may contain information (ie, are informative) about pathologists' work were selected for export from the LIS; the actual data selected for export from LISs were determined by the AABACUS development/implementation team (CCC, EET, HC, SLA). Once exported as either comma-separated value files or Excel files, these raw parameters were then imported into the AABACUS database where calculations were performed. There was no direct data mining. In addition, as shown in Figure 3, no modifications to the LIS were required and no change in the way in which the LISs were used was required. This approach is applicable to any LIS system that can generate reports and/or export data it collects through usual operations; no specific programming or reprogramming of the LIS is necessary.

\section{Identify}

'Identify' includes the identification of workload parameters and the identification of workload activities related to them. Workload parameters were either (1) raw parameters that in and of themselves were informative for pathologists' work (eg, number of original H\&E sections produced and therefore presumed to have been analyzed by the primary pathologist for that specimen) or (2) were calculated from raw parameters (eg, the workload parameter of 'number of actual blocks' was derived from subtracting the raw parameter of 'number of blocks canceled' from the raw parameter of 'number of blocks originally printed').

Workload activities were defined as the documentable activities performed by pathologists within the sphere of their clinical practice and were derived/ calculated from workload parameters. For example, the presence of the workload parameter 'original

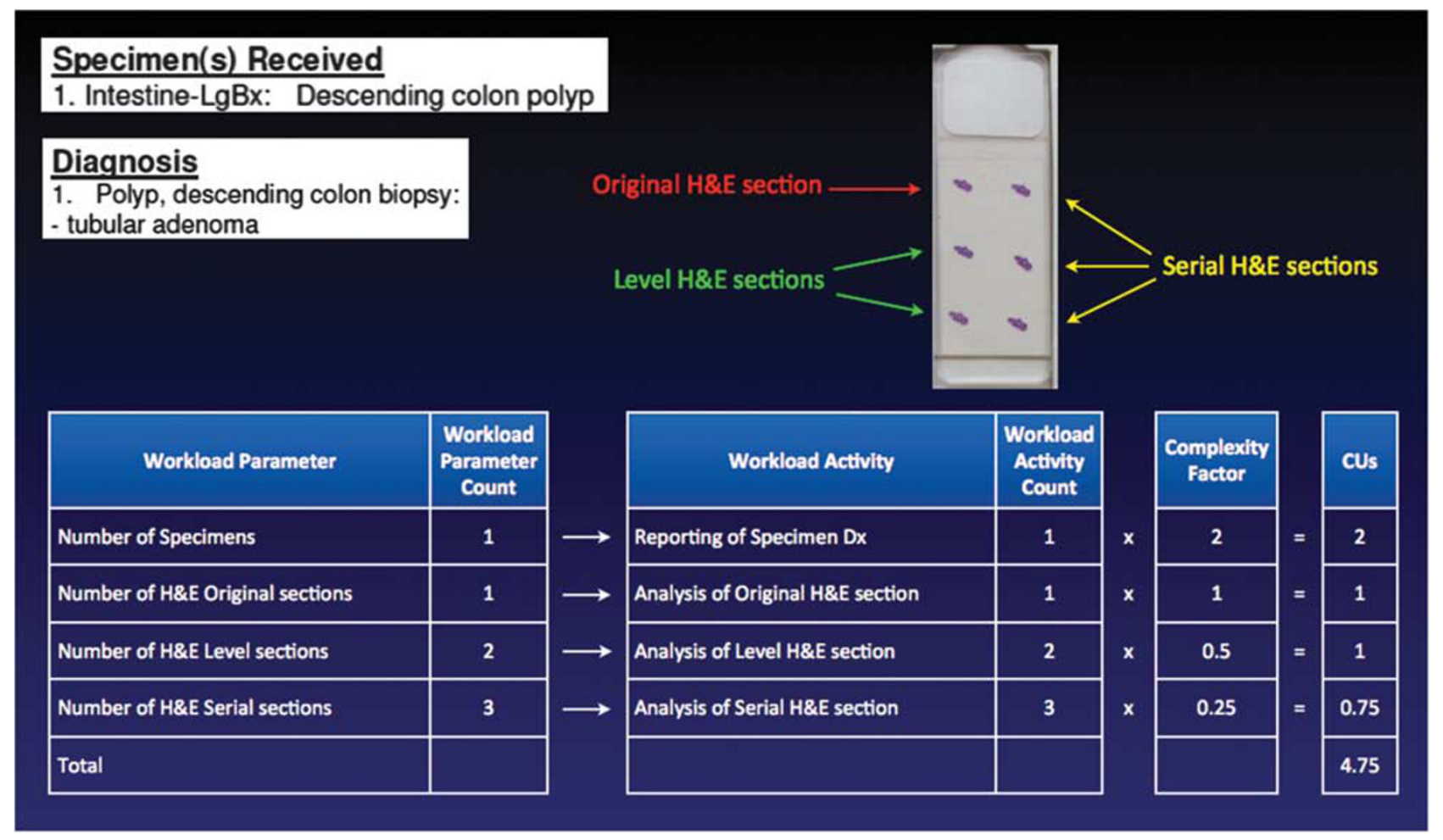

Figure 4 Colonic polyp with levels (tubular adenoma); an illustrated example of AABACUS in action. 


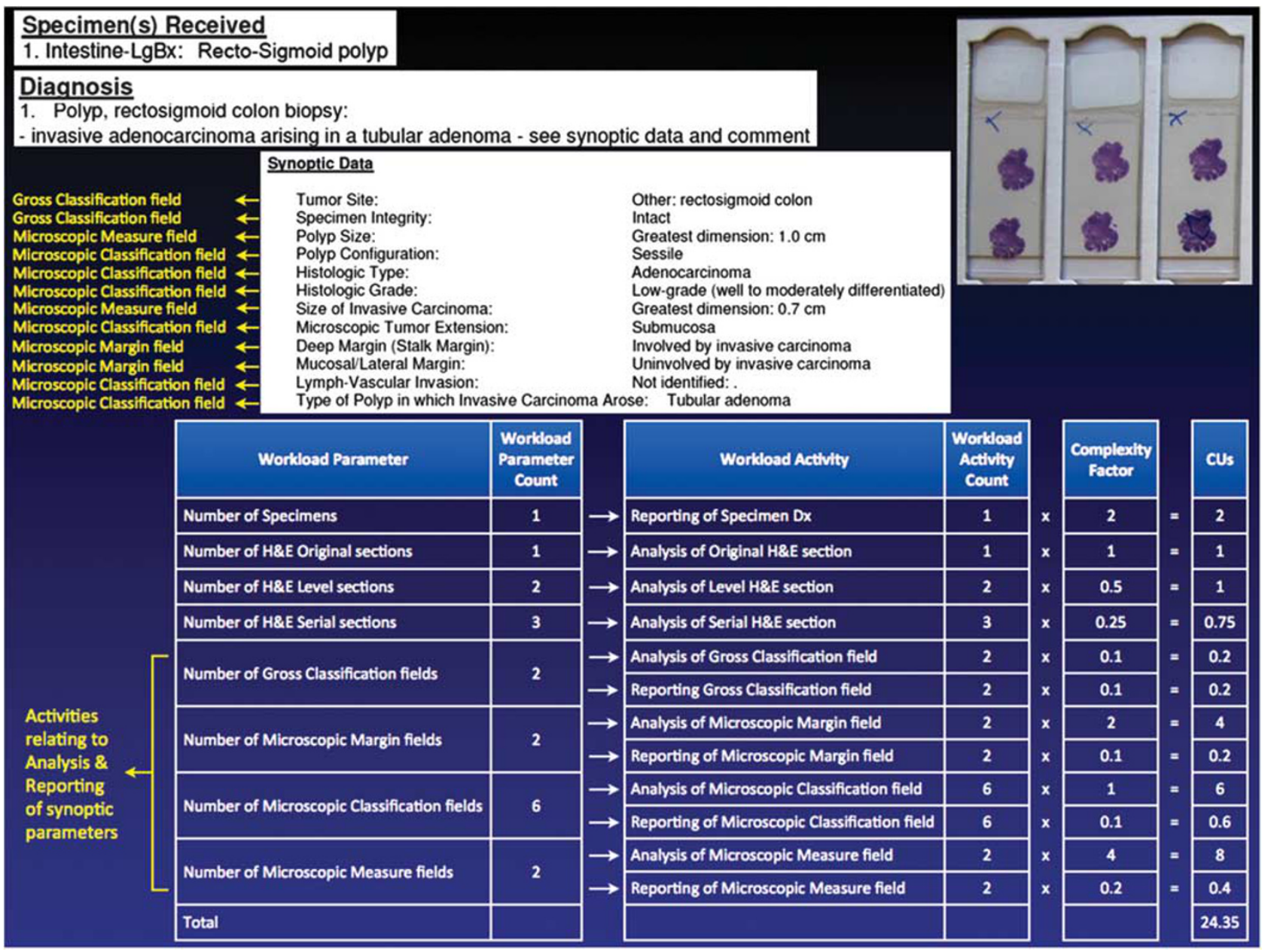

Figure 5 Colonic polyp with levels (adenocarcinoma... with synoptic data); an illustrated example of AABACUS in action.

H\&E section generated' was extrapolated to signal the occurrence of the workload activity 'analysis of an original H\&E section'. Some workload parameters were associated with only one workload activity, but some were associated with more than one (see Figures $4-7$ and Supplementary Figures S1-S9 for examples of this). We sorted each 'workload activity' into one of four categories: specimen acquisition activities, specimen handling activities, analysis activities, and reporting activities (Figure 2); QA activities were also accounted for primarily under the analysis and reporting categories of activities.

Specimen acquisition activities include the performance of biopsy procedures, such as FNA biopsies, bone marrows, and other specimens that are collected by a pathologist.

Specimen handling activities include the direct handling of wet specimens by pathologists including grossing/blocking of surgical pathology specimens during intraoperative frozen sections and during routine grossing, the handling of fresh tissue for submission for 'lymphoma protocols', the generation of direct smears by pathologists during onsite assessments at FNA clinics, and so on.
Analysis activities are those required to determine the correct diagnosis and relevant theranostic information. Credit was given to gross and microscopic examination, number and types of slides examined for each case, morphologic parameters that must be assessed for subsequent reporting as part of clinical synoptic reporting templates, and special studies of all types performed on a given case, such as flow cytometry, immunohistochemistry, electron microscopy, FISH, molecular testing, and all other types of novel testing. Our pathology LIS uses College of American Pathologists (CAP) synoptic sheets for cancer reporting that we have analyzed for clinical activities and scored. For example, determining the presence or absence of angioinvasion is a morphologic component of evaluation; measurement of tumor size, depth of invasion, or distance to a resection margin are absolute measurements; mitotic counts are categorized as a high-power counting activity, whereas 'percent involvement' is considered a relative measurement. We also created synoptic reporting templates in a manner similar to the cancer synoptic sheets for noncancer evaluations where expert 


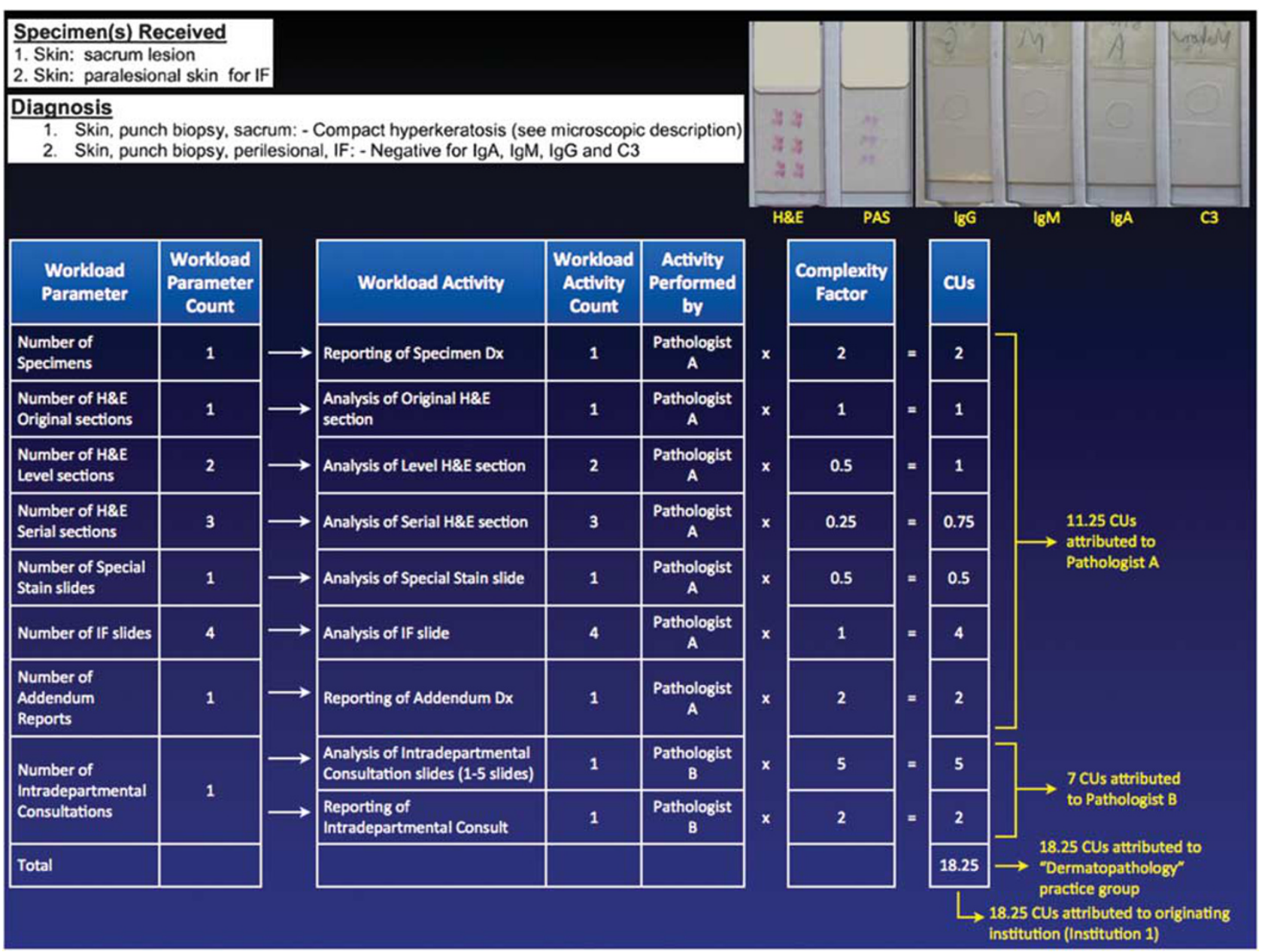

Figure 6 Skin biopsy with levels, immunofluorescence, special stain, and intradepartmental consultation (ie, second opinion before sign-out); an illustrated example of AABACUS in action.

groups recommended it for purposes of both standardized reporting of clinically relevant parameters and proper capture of the work required to analyze and report those parameters (eg, a nontransplant liver biopsy reporting template as shown in Supplementary Figure S9).

Reporting activities include activities performed in order to document the information acquired/ generated during analysis activities in a diagnostic medical report, and includes both nonsynoptic (ie, traditional free text 'diagnoses') and synoptic components. Examples include: the actual reporting of each specimen within a final report, the reporting of intraoperative frozen section consultations, the reporting of molecular/cytogenetic testing, the selection and actual filling out of synoptic worksheets, activities after sign-out such as issuing addenda, and completion of a theranostic report.

QA activities involve both case sharing before sign-out as well as reviews after sign-out; these also include review of previous pathology reports and/or slides for rounds/tumor boards, review of clinical notes from the patient chart, and review of radiologic reports and/or radiologic images. The vast majority of pathologists perform QA routinely, when confirming previously issued intraoperative consultation diagnoses at the time of case sign-out, and when sharing a difficult case with a colleague (ie, intradepartmental consultation before sign-out). We document such activities in our LIS with a series of discrete data fields that include the number of slides reviewed. These activities were also allocated workload units that were ascribed to the pathologist performing the activities.

Although it is entirely feasible to capture workload parameters and/or the workload activities manually, we have utilized data capture by our LISs (see Figure 3). The advantage of using LIS-captured parameters is that it is automated and as such saves time, is more reliable than manual entry, and is more objective because no interpretation is required.

\section{Count}

'Count' pertains to the counting of the number of times each identified workload activity occurs. 


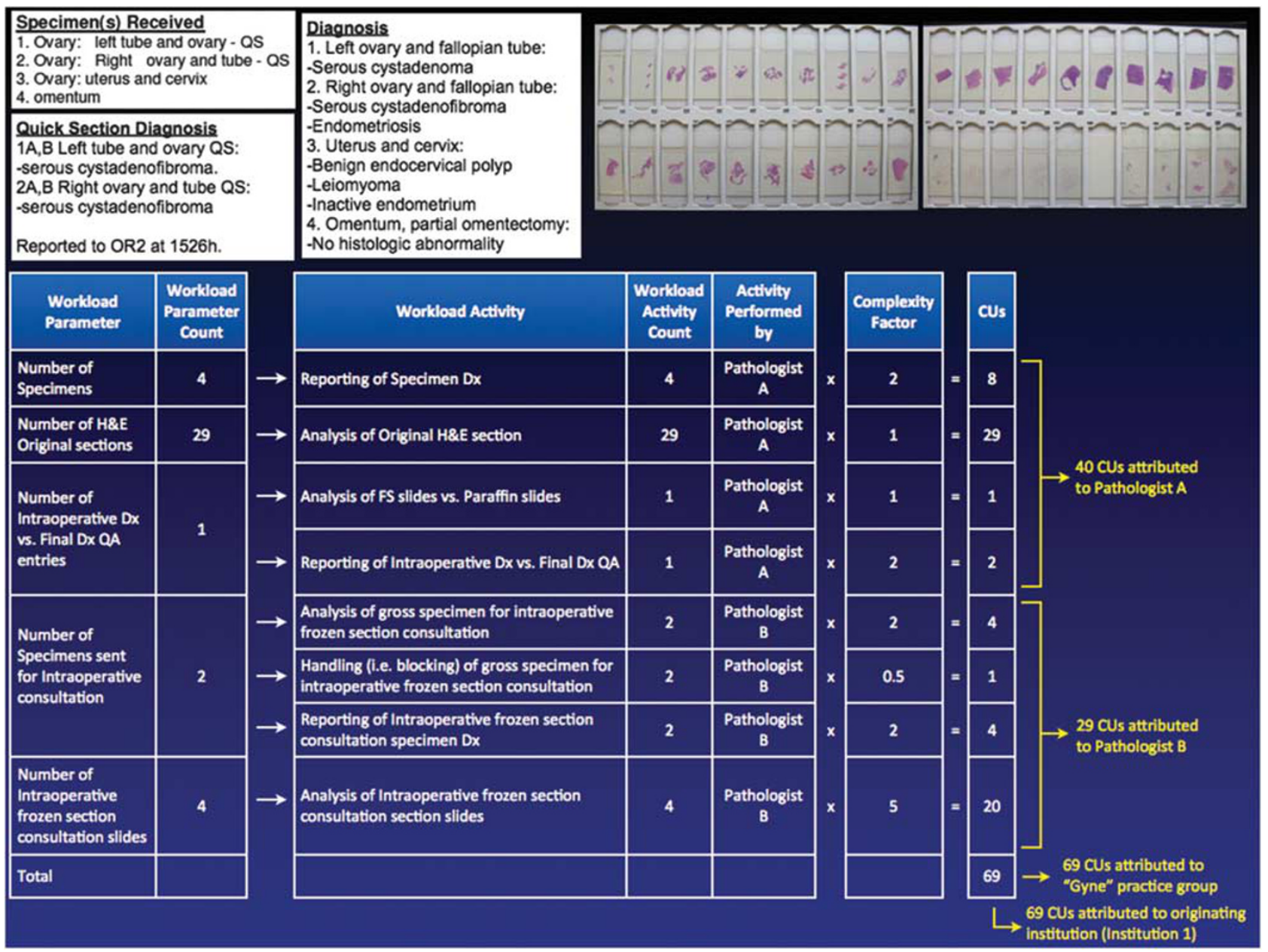

Figure 7 Total abdominal hysterectomy and bilateral salpingo-oophorectomy with intraoperative frozen section consultation; an illustrated example of AABACUS in action.

\section{Score}

'Score' represents the conversion of workload activity counts into complexity units (CUs), usually by 'complexity factor' multiplication (see illustrated example in Figure 4). The 'complexity factor' for various workload activities was assigned by consultation and input from a group of 50 pathologists to ensure that all subspecialty practice perspectives were obtained. Sample calculations are shown in Figures 4-7 and Supplementary Figures S1-S9.

\section{Attribution and Filtering}

Once scored, CUs were then attributed to institutional sites (ie, hospitals), practice groups, and individual pathologists. Some parameters that allow for such attribution are already derived by the LIS (eg, main sign-out pathologist for a case). Some, not directly available in the LISs, were attributed in AABACUS to allow for such assessment. When multiple pathologists worked on one case, they all received credit for work performed if documented in the LIS (eg, frozen sections, grossing, QA, addenda). Representative examples of such attribution including cases for which there were contributions from multiple pathologists are shown in Figures 6 and 7 . 'Filter' pertains to presentation of already calculated results by institution, practice site group, and/or individual pathologist.

\section{Assess Filtered Results}

'Assess filtered results' includes, but is not limited to, overall staffing assessment, targeted staffing assessment based on subspecialty practice needs, utilization assessment, and/or resource allocation assessment (Figure 3).

\section{Application of AABACUS to Practice Setting}

The model was applied to the LIS-documented clinical work being performed by pathologists within a complex multi-institution structure that includes four institutions, two of which are multisite, 
Table 1 Institutional profiles

\begin{tabular}{lccc}
\hline & Institution 1 & Institution 2 & Institution 3 \\
\hline Size (number of beds) & Large (600) & Small (167) & Small (167) \\
Cancer center & Yes & Yes & No \\
Transplant center & No & No (773) & Yos \\
Clinical responsibilities & Yes & Yes & Yes \\
Educational responsibilities & Yes & No & No \\
Research responsibilities & No & No & Yos \\
\end{tabular}

three of which include cancer centers, and one of which is an academic quaternary-care referral center (Table 1). In this multi-institutional setting, pathologists affiliated with any of the institutions may perform work on specimens that originate from any of the other institutions. For example, a pathologist whose primary affiliation is with institution 4 may be the primary sign-out pathologist for specimens that originate from institutions $1,2,3$, or 4 .

\section{Results}

\section{Assessment of Overall Staffing Levels}

To examine overall staffing levels, we used a relative benchmark, the 'clinical service full-time equivalent (csFTE)', that represents the work of a full-time pathologist in a nonacademic institution who carries out clinical diagnostic work with no responsibilities for teaching or research activities. In order to define one csFTE, we took the total number of CUs generated for institution 2, which is a nonacademic institution (as shown in Table 1), and divided that by the total number of funded positions allotted to that institution (Figure 8).

We identified that, if using institution 2 as the relative benchmark, $\sim 40000$ CUs would be the appropriate target clinical workload of a csFTE per annum (see Discussion below). Using institution 2 as the benchmark institution, the number of csFTEs needed to perform the clinical service load of each target institution was calculated and plotted along with the current number of csFTEs funded for each institution. The data suggest that 12.6, 4.3, and 41.5 csFTEs are required for institutions 1,3 , and 4 , respectively, if the clinical workload per pathologist for institution 2 is used as the relative benchmark. However, as institution 4 has requirements for teaching and/or research by pathologists, there is a need for such an academic center to provide nonclinical service-related requirements for teaching and research. The data can be used to negotiate an institutional vision with respect to the importance of academic output by pathologists. Once determined, the education and/or research workload of each pathologist must be determined in conjunction with clinical workload.

\section{Assessment of Subspecialty Staffing Levels}

To examine the distribution of clinical service among 14 established practice groups (Breast, Cardiovascular, Cytopathology, Dermatopathology, Endocrine, Gastrointestinal, Gynecologic, Head and Neck, Hematopathology, Hepatobiliary, Neuropathology, Renal, Thoracic, and Urologic), the total number of CUs for each practice group across all institutions is shown in Figure 9. For example, if a full or partial vacancy arises, the data from Figure 9, in conjunction with an assessment of the current subspecialty expertise available, can be used to recruit pathologists with particular subspecialty expertise as necessary.

It should be noted that Autopsy pathology is not included as a practice group since pathologists of any practice group may perform autopsies in this organization where there is no forensic autopsy work. Therefore, CUs associated with autopsy activities contribute only to institutional workload and individual-based workload, but not to practice group workload. In an institution where autopsy pathology is devolved to specialists, such as forensic or academic autopsy pathologists, this information can be categorized as a separate group.

\section{Assessment of Staffing Distribution for Clinical Service Coverage}

The proper distribution of available staff in providing clinical service coverage can be an important determinant in managing pathologist clinical workload. As an example of this, in Figure 10 we show the relative breakdown of clinical services provided by our hematopathologist group. In determining a hematopathologist clinical service schedule, the data would suggest, for example, that the ratio of staffing should be 4:1 for pathologists performing bone marrows/peripheral blood smears/body fluids/ hemoglobin electrophoresis versus those analyzing lymph nodes alone. Properly planned and distributed clinical service schedules facilitate reasonable clinical loads and achievable turnaround times.

\section{Assessment of Clinical Workload among FTE Groups}

In academic departments, individual pathologists require or are promised protected time for research 
a

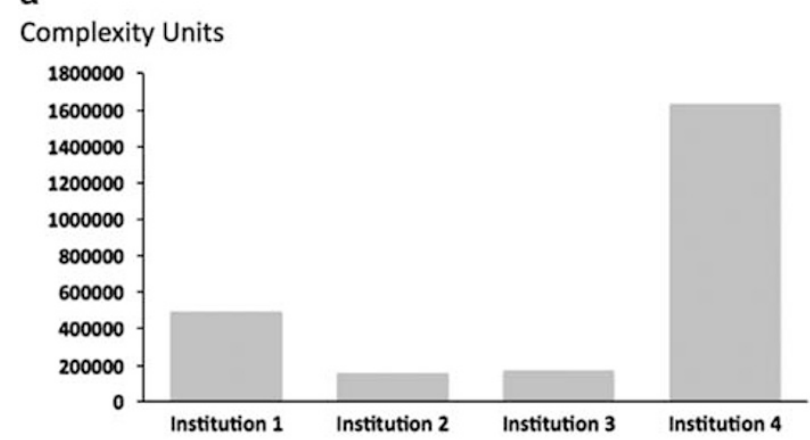

b

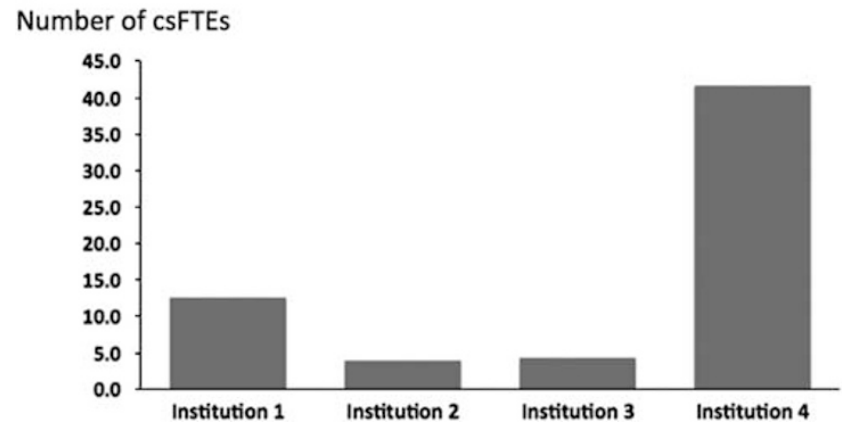

Figure 8 Assessment of overall staffing levels for each of the four institutions using relative benchmarking. Light grey bars represent the total number of CUs for each institution (a). Dark grey bars represent the number of clinical service full-time equivalents (csFTEs) needed if institution 2 is the benchmark institution (b).

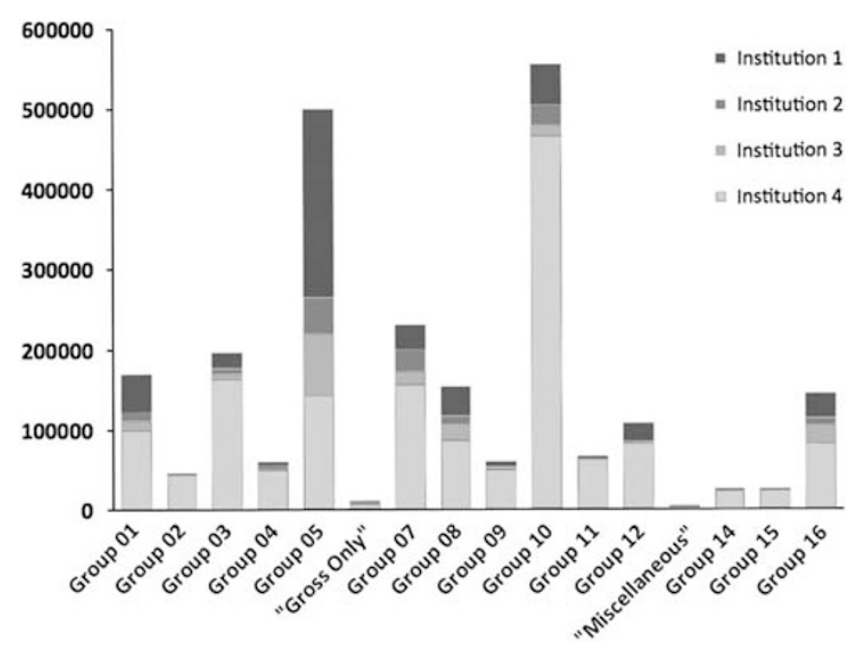

Figure 9 Total CUs by practice group across all institutions for LIS-capturable clinical work for 2012.

and teaching activities. The measures of academic productivity are beyond the scope of this study. However, this model helps define how much clinical responsibility can be assigned to academic staff once the decision has been made about what portion of a FTE will be attributed to such an individual. We therefore compared the CUs of three

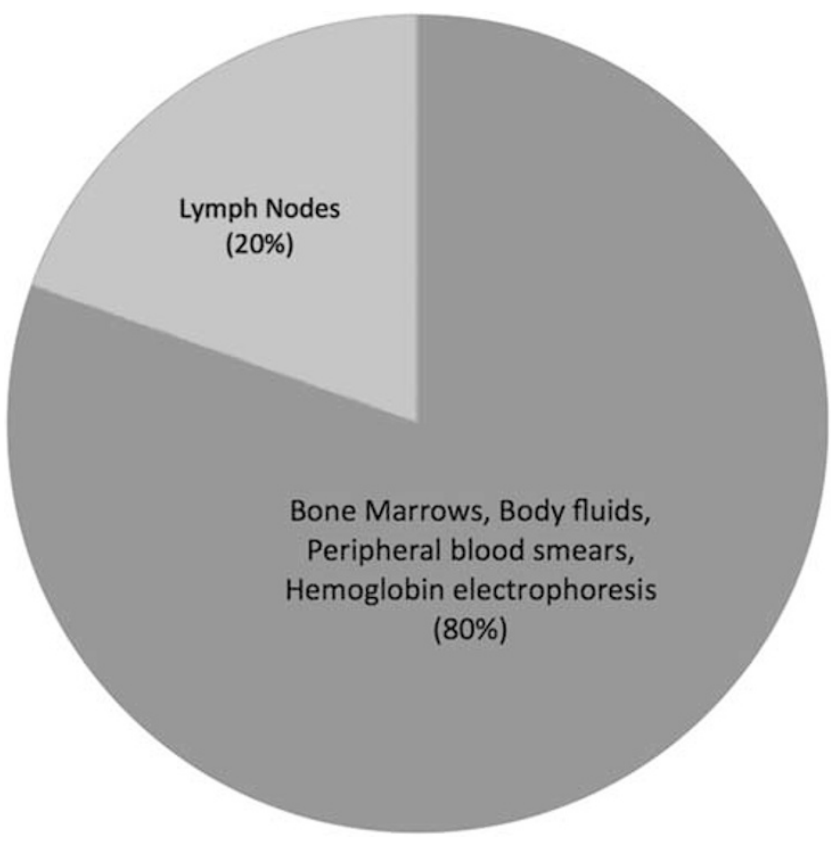

Figure 10 Distribution of CUs for the hematopathology practice group for assessment of staff for clinical service coverage.

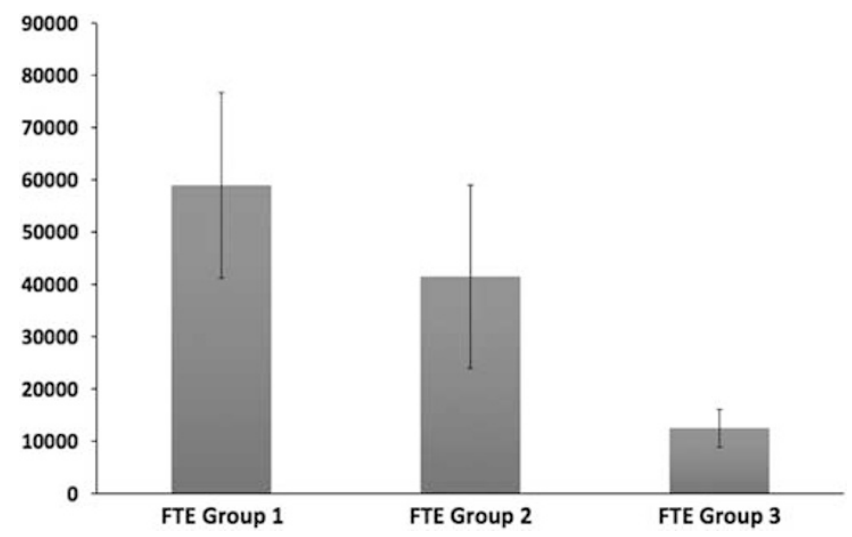

Figure 11 Average CUs per full-time equivalent (FTE) group for $2012 \pm$ s.d. FTE group 1 represents pathologists with no significant academic responsibilities. FTE group 2 represents pathologists with teaching and research responsibilities. FTE group 3 represents clinician scientists with significant expectation for research output.

groups of individuals within our various departments across all institutions. Group 1 consists of pathologists who have no significant academic responsibilities. Group 2 consists of clinical educators or clinical investigators who are expected to publish and teach as well as carrying out diagnostic activities. Group 3 represents clinician-scientists who are committed to $75-80 \%$ protected time for grant-funded research. Figure 11 shows the CUs of these three groups of individuals across all four institutions, illustrating that this model can be used to allocate and oversee clinical activities in a multiprofile organization. 


\section{Comparison with RVUs}

In the United States, CPT (common procedural terminology) coding is used to determine reimbursement for pathology specimens. CPT codes are assigned based mainly on the specific specimen type (eg, a skin biopsy or colon resection) with some modification for the anticipated disease process in the specimen (ie, a rectum resected for tumor is given a higher CPT code than one resected for prolapse), although for many specimen types (such as skin biopsies or appendectomy specimens) the code is not modified regardless of the pathological findings. Size of the specimen is never taken into account for CPT coding purposes. Although originally created as part of Medicare reimbursement, the CPT coding system is now universally used for billing. CPT codes are converted to RVUs using a multipart calculation, including factors such as the geographical location of the pathologist, which is beyond the scope of this article. These RVUs are then used to determine compensation for pathology examination of a specimen and are also used as a tool for determining workload and hence staffing.

Although RVUs are used in the United States to designate complexity of cases, because there is low granularity in the system, use of RVUs as a surrogate measure of workload may underestimate or overestimate actual work done. Table 2 illustrates for several representative types of cases how CPTderived RVUs compare with AABACUS, which measures work done rather than work that is presumed to have been done (or not). The CPT code 88305 designates the same workload to specimens such as a tubular adenoma and a malignant melanoma. Because AABACUS allows for a much greater degree of granularity, it allows us to show that the work done for a typical tubular adenoma is much less than the work done for a malignant melanoma.

\section{Impact Analysis of Pathologist-Initiated Activity on CUs}

In order to evaluate the possibility that pathologist workload may be artificially 'inflated' by patholo- gist-initiated activities, we expressed the total CUs associated with pathologist-initiated activities as a proportion of the total CUs for all pathologist activities of the target group(s). We show the example of immunohistochemical stains. Figure 12a shows the total number of IHC stains by practice site group across all institutions. Figure 12b shows the total number of CUs associated with the analysis of the IHC stains by the total CUs for practice group 10, the hematopathology group, that 'orders' the highest numbers of IHC stains. The data show that despite the high numbers of IHC stains ordered by the hematopathology practice group, the clinical work associated with the analysis of IHC stains represents

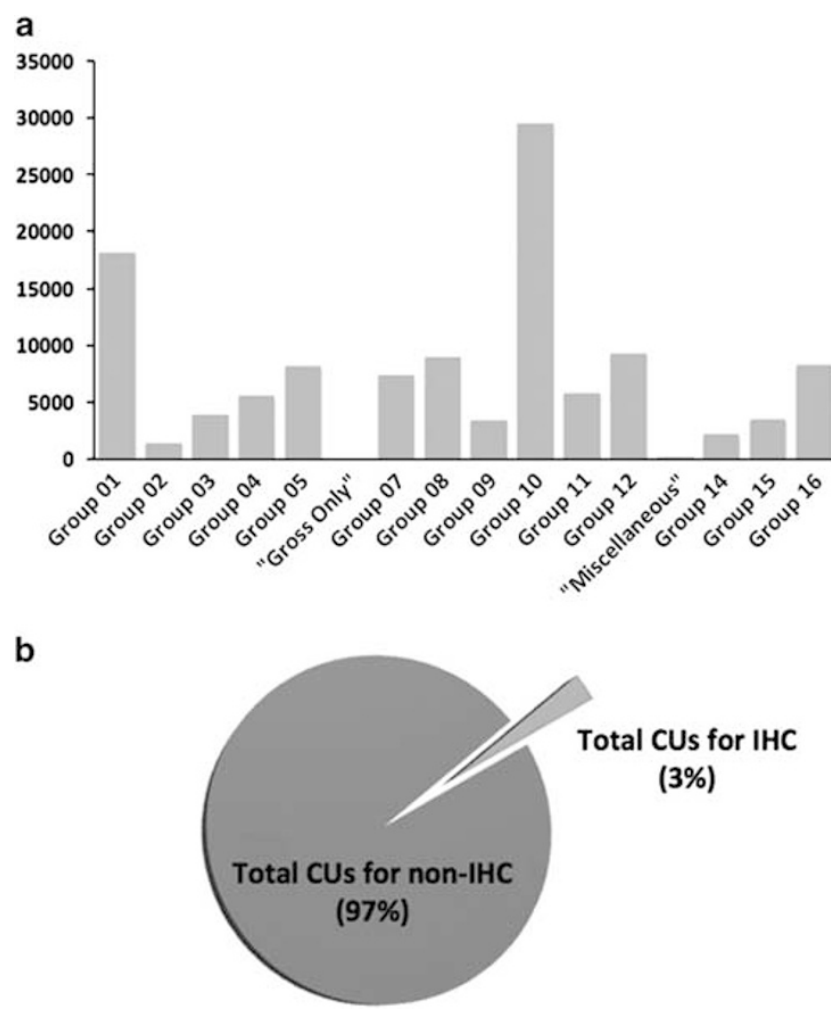

Figure 12 Number of immunohistochemical stains by practice group across all institutions for 2012 (a). Percentage of CUs credited for the analysis of immunohistochemical stains for the hematopathology practice group for 2012 (b).

Table 2 Summary comparison of RVUs and CUs

\begin{tabular}{|c|c|c|c|c|}
\hline Reference figure & Description & CPT code & $R V U s$ & CUs \\
\hline Figure 4 & Colonic polyp with levels (tubular adenoma) & 88305 & 0.75 & 4.75 \\
\hline Figure 5 & Colonic polyp with levels (adenocarcinoma...with synoptic data) & 88305 & 0.75 & 24.35 \\
\hline Supplementary Figure S1 & Skin biopsy with levels (intradermal nevus) & 88305 & 0.75 & 4.75 \\
\hline Supplementary Figure S2 & Skin biopsy with levels (melanoma) & 88305 & 0.75 & 36.95 \\
\hline Supplementary Figure S3 & Appendectomy (appendicitis) & 88304 & 0.22 & 5 \\
\hline Supplementary Figure S4 & Appendectomy (neuroendocrine tumor...with synoptic data) & 88304 & 0.22 & 36.9 \\
\hline Supplementary Figure S5 & Cholecystectomy (chronic cholecystitis) & 88304 & 0.22 & 4 \\
\hline Supplementary Figure S6 & Cholecystectomy (adenocarcinoma...with synoptic data) & 88304 & 0.22 & 35.4 \\
\hline Supplementary Figure S7 & Nephrectomy with $3.2 \mathrm{~cm}$ mass (oncocytoma) & 88307 & 1.59 & 9 \\
\hline Supplementary Figure S8 & Nephrectomy with $3 \mathrm{~cm}$ mass (renal cell carcinoma...with synoptic data) & 88307 & 1.59 & 38.1 \\
\hline Supplementary Figure S9 & Nontransplant liver biopsy (hepatitis C... with synoptic data) & 88307 & 1.59 & 39.4 \\
\hline
\end{tabular}




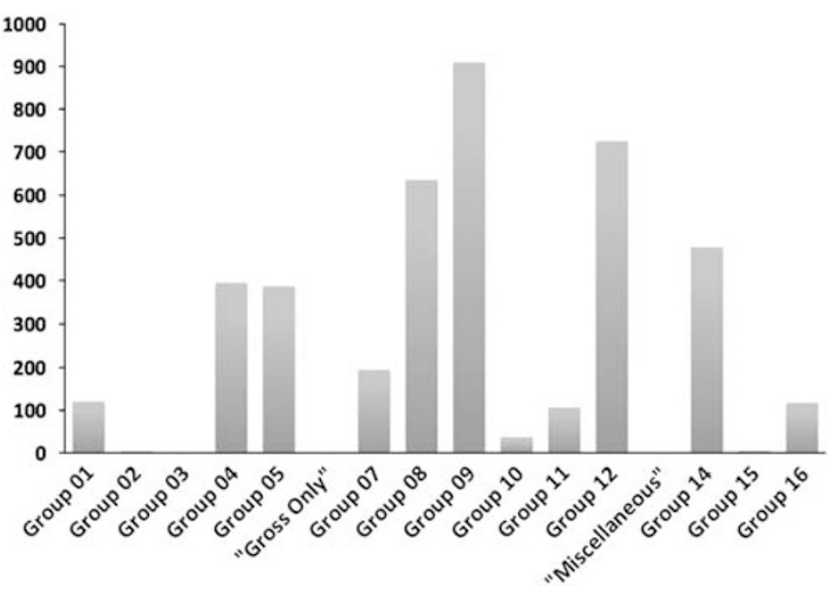

Figure 13 Number of frozen section slides by practice group for institution 4 for 2012 .

only $2.65 \%$ of that group's overall workload. This illustrates that the assignment of relative weightings to clinical activities under this model makes it difficult for total CUs to be skewed by pathologistinitiated activity.

\section{Utility for Non-Workload Applications: Utilization and Resource Allocation}

AABACUS can be used as an administrative tool to guide management decisions with respect to resource allocation and utilization. For example, if a new surgeon for practice group 08 were to be hired by institution 4 , we can use this model to help extrapolate the potential impact of this hire for pathology technical and professional intraoperative frozen section consultation services. Figure 13 shows the number of frozen section slides generated by cases of each practice group for institution 4 . This information, in conjunction with the number of practice group 08 surgeons that contributed to the generation of these cases, allows us to extrapolate the expected impact on intraoperative frozen section consultations with respect to technologist resources and equipment use.

\section{Discussion}

The principal difference between AABACUS and other workload models is that other models capture presumed work done and apply weighting to the 'specimen type', whereas AABACUS captures actual measurable work performed by pathologists and is activity based rather than specimen based. Because of this, AABACUS allows for a degree of granularity that better reflects actual work done by pathologists.

\section{Defining and Measuring Complexity}

Complexity is well recognized as a limiting factor in workload measurement tools. ${ }^{18}$ However, the problem with complexity is one of definitioncomplexity may refer to having to perform ancillary tests, seeking of additional opinions, establishing the presence of malignancy, and reviewing of a patient's complete medical history. For the purpose of pathologist workload measurement, we have identified two major types of complexities: procedural complexity and cognitive complexity.

Cognitive complexity can be described by the following phrase: 'This case was complex because I had to think really hard and integrate a lot of information together into a picture that makes sense' (eg, interpretation of morphology-based parameters, clinical history, literature searches, and so on). Although there is no system that can truly measure all aspects of cognitive complexity, AABACUS does allow for partial capture of cognitive complexity through two mechanisms: (1) documentation of certain activities, and (2) applying relative weightings to activities. For example, the documentation of reviews of previous pathology reports, clinical/ radiological data, slides from previous cases and so on, are captured as QA activities. In this way, AABACUS can give credit for activities such as having to look through clinical notes in order to 'piece together a hard case' and can apply a relative weighting to such an activity. However, what it cannot do is determine how difficult it was for the pathologist to understand/comprehend what was written in the notes of the clinical chart. There are no objective measurable outcomes for cognitive complexity and thus it is difficult to quantify. As such, all models that account for cognitive complexity (eg, those that apply relative weightings) accept the subjective nature of this exercise. Procedural complexity can be described by the phrase: 'This case was complex because I had to perform many tasks (mental and physical) in order to finish it'. Compared with cognitive complexity, procedural complexity is easier to capture and measure.

AABACUS measures procedural complexity through the identification and counting of clinical workload activities as described above. It partially addresses the concept of cognitive complexity by assigning relative weights (ie, via multiplication by a complexity factor) to the workload activities based on expert consensus. Considerations such as experience and expertise, both of which feed into the concepts of 'difficulty' and 'stress' and 'level of risk' and 'value to clinicians', are not specifically taken into account because they cannot be objectively measured. As such, the proper definition of the AABACUS model's base output unit, the CU, followed by benchmarking considerations, is paramount.

\section{Defining the CU}

The base unit of any workload measurement model is arbitrary. As AABACUS is activity based rather than specimen based, we chose an activity, and not a 
specimen, for our base unit. As the H\&E section is the lingua franca of morphology-based pathology practice, we designated one CU to the 'analysis of one H\&E-stained tissue section'. Similarly, all other activities were also assigned relative weights (ie, complexity factors) based on expert consensus. Considerations like 'effort', 'medico-legal risk', 'skill', 'experience', and 'judgment' did not factor into the CU. It must be stressed that 1 CU does not represent the actual amount of time it would take for a pathologist to perform an activity that was assigned $1 \mathrm{CU}$. For example, different H\&E sections require different amounts of time for analysis and different pathologists may require different amounts of time for the same H\&E sections depending on level of expertise/experience in a certain subspecialty fields, or even transient/unpredictable factors such as whether the analysis is performed in full health or on the time of day. Hence, unless we move to a pure time-docketing system, which would render all current models irrelevant, it would be impossible to account for all of the possible variations involved in this one activity. Therefore, $1 \mathrm{CU}$, rather than representing the actual amount of time for any specific activity designated as being worth $1 \mathrm{CU}$, instead represents an acceptable amount of time granted for any such activity.

Using again the example of 'analysis of an H\&E section' as a baseline activity (ie, an activity that was designated as representing $1 \mathrm{CU}$ ), we can ask: what is an 'acceptable' amount of time for this activity? The answer depends on the purpose of the question. There are two instances when a specific time value may be assigned to $1 \mathrm{CU}$. The first instance occurs when determining the complexity factors for the various AABACUS workload activities. Here, our expert consensus panel members assigned whatever amount of time they deemed to be appropriate for a baseline activity (eg, analysis of an 'average' H\&E section) and weighed other workload activities with which they had experience relative to that baseline activity. It did not matter what those time values were nor did it matter that many members had different time values in mind for the baseline activity; this is because the complexity factor is a ratio. Therefore, for the purpose of determining complexity factors by consensus, the specific time value assigned to a baseline activity such as 'analysis of an H\&E section' can be any time value that allows panel members to be comfortable and confident in weighing other workload activities relative to the baseline activity. In the design of AABACUS, this is the step where all workload activities were placed in relative standing to each other by expert consensus.

The second instance where a specific time value may be assigned to $1 \mathrm{CU}$ occurs when absolute benchmarking is used (see section below 'Benchmarking considerations: Absolute and Relative Approaches'). Here, when considering what is an 'acceptable' amount of time for a baseline activity (ie, $1 \mathrm{CU}$ ), the question of 'acceptable to whom?' becomes important. Although both pathologists and payers would undoubtedly support rationality and fairness, it is not difficult to understand that payers, because of fiscal pressures, may prefer to have as much work as possible being done by pathologists for any amount paid. For example, a payer may suggest that $1 \mathrm{CU}$ should be worth $5 \mathrm{~s}$ because that is more financially advantageous for them; pathologists may suggest that $1 \mathrm{CU}$ be worth $5 \mathrm{~min}$ because it allows for many contingencies including the incorporation of activities that may take time but cannot be captured by current models. At some point though, pathologists and payers within a particular remuneration system must meet at a mutually agreeable definition of what is considered reasonable and acceptable; this definition is an arbitrary decision and can be changed by different institutions as appropriate for their practice setting. As will be discussed in the 'Benchmarking' section below, we utilized absolute benchmarking for only one specific reason. For the purpose of absolute benchmarking, we made the decision that in our multisite, multi-institutional practice setting that encompasses both subspecialty and general signout, 1 CU be worth 2 min. Our ultimate decision was arbitrary, but for us, the evolutionary roots of our decision-making process originated in The American Board of Pathology and the Royal College of Physicians and Surgeons (Canada) examinations in Anatomical Pathology that 'assesses candidates' ability to practice Anatomical Pathology independently'. In these examinations, candidates have at least 2 min to analyze slides (almost always H\&Estained sections) for analysis of overall quality of tissue sectioning/staining and to provide the most appropriate diagnosis based on what is represented on that slide alone. This represents the average amount of time accepted by specialty boards for this activity (the analysis of one H\&E section), whether in reality, depending on individual circumstances on a section-by-section basis, more or less time may actually be required. Although $2 \mathrm{~min}$ may also appear to be arbitrarily selected by the Boards, we accepted that the Boards are governed by national experts and that the time allowed for such analysis of H\&E sections has stood the test of time and has not changed for many years. Therefore, our assignment of 2 min for $1 \mathrm{CU}$ for the purpose of absolute benchmarking while arbitrary was guided by standards set by North American pathology examination Boards. Most importantly though, when we applied ' 1 CU is 2 min' to our practice environment, it appears to accurately reflect our workload. If institutions wish to change this default, they can do so, depending on what is considered possible and/or fair/justifiable for their particular practice. However, it is important to note that to compare between completely different institutions, relative benchmarking must be used rather than absolute benchmarking where the definition of 1 CU being 
2 min (or any other unit of time) is not relevant (see 'Benchmarking considerations: Absolute and Relative Approaches' below).

\section{What Components of Pathologist Work Are Measured?}

AABACUS measures activities in clinical laboratories related to specimen acquisition, specimen handling, analysis by pathologists, and reporting the results of the analyses performed. Specifically, it focuses on 'LIS-capturable' pathologist activities related to the issuing of diagnostic medical reports, including related QA activities; this also includes the ability to capture situations where multiple pathologists perform activities for the same case. Although any pathologist activity that can be captured by and/or translated from LIS parameters may be tracked using AABACUS, we have chosen to focus on clinical activities only (ie, clinical workload activities); the tool can be used to also account for the component of teaching activities that are performed using clinical cases if the presence of the trainee is documented in the LIS (ie, educational workload activities). In addition, administrative activity not specifically related to the generation of a diagnostic medical report is also not measured (ie, administrative workload activities). Other additional activities, such as attendance at multidisciplinary cancer conferences, can be captured as clinical workload when QA documentation of case preparation for those rounds is entered in the LIS. Depending on the institution, specimen-handling activities may be delegated to pathologist assistants, technologists, and trainees. The workload attributed to the pathologist of record for specimen handling activities will vary depending on the circumstances of the situation. For accountability purposes, most practices require that it be clearly documented who performed the various activities relating to specimen handling (eg, who grossed/ blocked a specimen, who embedded, who cut any particular block, who loaded the stainer, who delivered the slides, and who filed what material in the clinical archives). In this way, credit can be assigned to a pathologist if he/she performed any specimen-handling activities.

\section{Professional Contribution to AABACUS}

Active and on-going pathologist involvement is fundamental to the validity and cogency of AABACUS. When first being set up, pathologist involvement is required for the valid translation of LIS raw parameters into workload activities and for the proper initial relative weightings of those activities. Once set up, on-going pathologist involvement is required to ensure that changes in clinical practice are properly reflected in workload activities as well as in any adjustments to the relative weightings of assigned complexity factors. This ongoing peer review is also an important mechanism for monitoring the degree to which workload data may be influenced by pathologist-initiated activity (eg, ordering deeper levels, immunohistochemical stains; see Figure 12). The necessity of ongoing pathologist involvement in the administration of AABACUS is further discussed below (see section 'Future Adaptability and Backwards Compatibility').

\section{Benchmarking Considerations: Absolute and Relative Approaches}

Absolute benchmarking. Although the absolute approach to benchmarking requires more assumptions than the relative approach and was not our preferred method for benchmarking, it is useful for protecting pathologists from 'right shifts' in workload (ie, where a payer dictates that everyone just has to do more work for the same or less remuneration; in such situations, relative benchmarks would actually remain unchanged). Assuming an individual pathologist takes 6 weeks per annum for vacation and/or CME activities, and that a 'full work week' consists of $40 \mathrm{~h}$, this would provide 1920 working hours or 115200 min per year. If we further assume that on average, $1 \mathrm{CU}$ is worth $2 \mathrm{~min}$ of pathologist time, then one could expect that $1 \mathrm{csFTE}$ would not exceed 57600 CUs. This would be on the assumption that '8-h' days are entirely filled with nothing except work that is directly linked to 'LIS-capturable' activities with no breaks or interruptions of any kind. However, it must be acknowledged that breaks and interruptions are realities of human life and also that a csFTE is usually required to carry out activities that are not linked directly to LIS-capturable activities and therefore are not identified in CU calculations. Such activities may include time spent contacting and talking with clinicians, reading and responding to emails/phone messages, and speaking with patients. It therefore must be expected that in most pathology laboratories, the approximate time accounted for by total CUs would not total $8 \mathrm{~h}$ per day.

Relative benchmarking. Here, to define one csFTE, the total number of CUs generated for LIS-capturable clinical workload activities for a selected benchmark institution for a selected time period is divided by the total number of funded clinical service positions for that institution. The resultant number of CUs represents a relative benchmark for the amount of work carried by $1 \mathrm{csFTE}$ in the system being evaluated. The advantage of relative benchmarking is that it represents the current state of reality for the time period selected and it is able to accommodate adjustments to the model. For example, if it were decided that the relative weights of one or some of the clinical workload activities should be adjusted, then this tool allows for the rescoring of previously identified and counted activities. Relative bench- 
marks would then be automatically adjusted as well. The disadvantage of relative benchmarking is that the selection of what is being benchmarked against must be made with care. In our multi-institutional setting, we chose institution 2 as our benchmark institution because it is a clinical cancer center with no teaching or research responsibilities. Thus, the 'clinical workload' of a pathologist in that setting was used as a relative benchmark for other pathologists in our system. Interestingly, when we took the total number of CUs generated by all institutions and divided it by the total number of FTEs (not csFTEs) allotted across all institutions, the number of CUs per FTE for the 2012 calendar year was 41000 . This suggests that our choice of benchmark institution was not unreasonable (see Figure 8).

\section{Applications of the AABACUS Model as a Decision Support Tool in Laboratory Management}

The most valuable aspect of the AABACUS model is that it allows for examination of data for multiple purposes. AABACUS can be applied to support staffing models. It is suitable for single and multisite institutions, as well as subspecialty and generalist practices. Institutional comparisons of CUs allow for overall manpower planning (Figure 8). Practice group comparisons of CUs allow for targeted recruitment in order to ensure that expertise will be available in the required areas (Figure 9). Individual group assessments of CUs permit titration of clinical activity versus other activities to ensure that those with academic commitments are carrying clinical loads appropriate to allow them to achieve such goals (Figure 11). AABACUS also showed that there is insufficient funding for FTE group 1 compared with the benchmark institution (60000 CUs vs 40000 CUs). Similarly, but to a lesser extent, there is also underfunding for FTE group 2 (Figure 11).

In the era of mergers and acquisitions, it has become a challenge to integrate staff from different institutions with different cultures into newly formed health-care partnership systems. The AABACUS model can be used to evaluate the work in multisite institutions to ensure that staff can reliably compare their workload and corresponding compensation to ensure fairness and transparency. In this situation, not all activities may be performed in the same institution; multisite models frequently allow redistribution of specimens/activities based on pooling of resources or expertise. Using the AABACUS model, if more than one person performs activities on a given specimen, each contributor can receive credit based on the activities performed; in contrast, weighting of specimens assumes a bundle of activities that likely accompany each type of specimen and attributes it to the pathologist of record for that particular specimen. Thus, the AABACUS model allows for a fair and equitable method of promoting collaboration that can result in a comprehensive clinical report for a patient and clinician, while still recognizing the input of specialists in providing second opinions before sign-out and in the interpretation of data from various technologies such as flow cytometry, cytogenetics, and molecular diagnostics.

\section{Future Adaptability and Backwards Compatibility}

Changes in clinical practice that result in significant increases or, on occasion, decreases in workload may remain unacknowledged by administration/ management if not also accompanied by concomitant changes in the number of cases, the number of specimen parts, or the specimen types. For this reason, a case-based metric (ie, number of cases) or any other indirect model rooted in categorization by specimen type may be less informative. As AABACUS is activity based, new clinically relevant activities may be added as necessary; this versatility allows it to remain truly reflective of dynamic clinical practice. To sustain its value, this model, like any other, must be monitored to stay reflective of changes in practice that will alter clinical workload activity. Moreover, the relative values of specific activities can and should change with changes in technology.

Because AABACUS collects workload activity counts before applying weightings (ie, complexity factors) to arrive at workload activity scores, it is possible to quickly and retroactively apply different complexity factors in calculating CUs. In other words, the complexity factors do not make the model and can be adjusted by expert consensus panels as required and/or as changes occur in clinical practice that affect pathologist workload. The membership of such panels will be determined by the intended scope of comparison. For example, a single-site institution may choose only members from its own pathology department, whereas a multisite institution may choose members from some or all of its sites. If multiple multisite institutions wanted to compare with each other, then those institutions can strike a mutually agreed upon panel of members to review (and/or adjust if desired) complexity factors of captured activities. Once agreed, scoring could be applied retroactively and relative benchmarking used for comparative analyses.

\section{Comparison with Other Workload Measurement Systems}

If workload measurement models are rooted in a specimen-type approach, which lacks the granularity required to enable measurement of actual procedural complexity, then they do not automatically capture increasing or decreasing complexity linked to work performed as dictated by changing clinical needs. Models that assign relative weights that are based on billing codes are vulnerable to 
shifts in economic and political status as they are not designed to measure work done, but rather what a payer is willing to pay at any specific time or location (eg, country, state, HMO). This means that the 'work performed' may artificially decrease or increase with the fluctuating price that is attached to that work.

AABACUS captures actual activities performed; it does not presume that a bundle of activities associated with specific specimen types will possibly be performed. Meijer et $a l^{19}$ have proposed an activity-based model to measure pathologist workload. They sought to more accurately calculate the time required to handle the gross and microscopic examination for specific specimen types but still assigned workload credit by specimen type on a prospective basis. AABACUS captures activities regardless of specimen type; in fact, although specimen type may be utilized during data analysis if so desired, it does not factor into actual CU calculations.

A major limitation in measuring workload by specimen type is that two of the 'same' specimens as defined either clinically and/or by gross visual inspection may have different findings on histologic analysis that require different informational parameters to be included in the diagnostic medical report. Although there are models that acknowledge the additional work required for completing a synoptic reporting template, ${ }^{10,14}$ AABACUS additionally gives credit to information parameters within synoptic template fields that must first be assessed (ie, as an analysis activity) and then documented in the diagnostic medical report (ie, as a reporting activity). This is in recognition of the fact that different informational parameters are associated with different types of analysis activities (eg, identification, counting, measuring, and so on).

Unlike models that require the entry/recording of workload-specific codes/data, AABACUS bases workload calculations on data that are already collected by LISs as a part of usual clinical workflow. Hence, although it is possible to collect workload related data manually, this feature of AABACUS was primarily conceived to work with data that are exported from LISs. AABACUS does not require any additional work by pathologists in order to capture workload activities.

\section{Conclusion}

AABACUS is a new activity-based pathologist workload measurement model that is objective, can be automated through the use of exported data from one or more laboratory information systems, is reproducible, backwards compatible, future adaptable, and is applicable across different laboratory medicine disciplines. It offers a method of obtaining an accurate capture of measurable pathologist clinical workload activities that cannot be easily skewed by pathologist-initiated activity. Because it is not based on any existing billing model, it offers stability in the face of changing economic and political tides, and is therefore useful as a decision support tool in laboratory management. AABACUS is suitable for monitoring pathologist clinical workload across different institutional practice profiles with applications to the assessment of overall and targeted staffing needs as well as utilization analyses for resource allocation.

\section{Disclosure/conflict of interest}

The authors declare no conflict of interest.

\section{References}

1 Baskovich BW, Allan RW. Web-based synoptic reporting for cancer checklists. J Pathol Inform 2011;2:16.

2 Beastall GH. The modernisation of pathology and laboratory medicine in the UK: networking into the future. Clin Biochem Rev 2008;29:3-10.

3 Louis DN, HWt Virgin, Asa SL. 'Next-generation' pathology and laboratory medicine. Arch Pathol Lab Med 2011;135:1531-1532.

4 Srigley JR, McGowan T, Maclean A, et al. Standardized synoptic cancer pathology reporting: a populationbased approach. J Surg Oncol 2009;99:517-524.

5 Black-Schaffer WS, Young RH, Harris NL. Subspecialization of surgical pathology at the Massachusetts General Hospital. Am J Clin Pathol 1996;106: S33-S42.

6 Groppi DE, Alexis CE, Sugrue CF, et al. Consolidation of the North Shore-LIJ Health System anatomic pathology services: the challenge of subspecialization, operations, quality management, staffing, and education. Am J Clin Pathol 2013;140:20-30.

7 Martin SA, Styer PE. Assessing performance, productivity, and staffing needs in pathology groups: observations from the College of American Pathologists PathFocus pathology practice activity and staffing program. Arch Pathol Lab Med 2006;130: 1263-1268.

8 Sanders DS, Carr RA, Stores OP, et al. Subspecialisation in cellular pathology in the DGH setting: the Warwick experience. J Clin Pathol 2006;59:884-886.

9 Trotter MJ, Larsen ET, Tait N, et al. Time study of clinical and nonclinical workload in pathology and laboratory medicine. Am J Clin Pathol 2009;131: 759-767.

10 Canadian Association of PathologistsCanadian Association of Pathologists, Manpower Committee, Update 2011. 2011. Available from http://www.cap-acp.org/ cmsUploads/CAP/File/CAP manpower 2011 update FINAL_2_June_2011.pdf (Accessed 30 July 2013).

11 Carr RA, Sanders DS, Stores OP, et al. The Warwick system of prospective workload allocation in cellular pathology-an aid to subspecialisation: a comparison with the Royal College of Pathologists' system. J Clin Pathol 2006;59:835-839.

12 Haber SL. Kaiser Permanente. An insider's view of the practice of pathology in an HMO hospital-based 
multispecialty group. Arch Pathol Lab Med 1995;119: 646-649.

13 Hsiao WC, Braun P, Dunn D, et al. Resource-based relative values. An overview. JAMA 1988;260: 2347-2353.

14 Path2Quality. Work2Quality: Guidelines for Workload Measurement in Pathology Professional Practices, version 1.2. 2012. Available from http://www.capacp.org/cmsUploads/CAP/File/Work2Quality_Proposal_-_September_16_2012.pdf (Accessed 30 August 2013).

15 Suvarna SK, Kay MS. KU activity: a method for calculating histopathologists' workloads. J Clin Pathol 1998;51:530-534.
16 The Royal College of PathologistsGuidelines on staffing and workload for histopathology and cytopathology departments, 3rd edn 2012. Available from http://www. rcpath.org/workforce/workforce-planning/workloaddocuments (Accessed 20 July 2013).

17 Goodson JD. Unintended consequences of resourcebased relative value scale reimbursement. JAMA 2007;298:2308-2310.

18 Winter S, Cox GJ, Corbridge R, et al. Effects of clinical service reorganisation on cellular pathology workload. J Clin Pathol 2004;57:22-26.

19 Meijer GA, Oudejans JJ, Koevoets JJ, et al. Activitybased differentiation of pathologists' workload in surgical pathology. Virchows Arch 2009;454:623-628.

Supplementary Information accompanies the paper on Modern Pathology website (http://www.nature.com/ modpathol) 